\title{
Receding-Horizon Control of Distributed Generation to Correct Voltage or Thermal Violations and Track Desired Schedules
}

\author{
Hamid Soleimani Bidgoli \\ h.soleimani@ulg.ac.be
}

\author{
Mevludin Glavic \\ mevludin.glavic@ulg.ac.be
}

\author{
Thierry Van Cutsem \\ t.vancutsem@ulg.ac.be
}

\begin{abstract}
This paper presents a real-time, centralized control system acting on the active and reactive powers of distributed generators when the network experiences voltage and/or thermal limits violation. The control resorts on multi-step recedinghorizon optimization. The objective is to minimize the deviations of Dispersed Generation Units (DGU) active and reactive powers from reference values. The reactive power corrections have priority over the active ones. Furthermore, the formulation is such that DGU powers are restored to their desired schedule as soon as operating conditions allow doing so. Three modes of operation of the proposed controller are presented, involving dispatchable units as well as DGUs operated to track maximum power output. The effectiveness of the proposed control is illustrated through detailed simulations of a 75-bus, 11-kV system hosting 22 DGUs.
\end{abstract}

Index Terms-active distribution networks, corrective control, receding-horizon control, multi-step constrained optimization.

\section{INTRODUCTION}

The environmental concerns are leading to a progressive growth of renewable energy sources connected to distribution systems. This development along with the intermittent nature of renewables is expected to create operational problems, mainly over- or under-voltage and/or thermal overload. The problem can be partly handled at the operational planning stage [1], [2]. However, in real-time, the system still can be driven to insecure situations, for instance due to unforeseen events. To deal with this issue, the Distribution System Operator (DSO) should install measurement devices to monitor the system, and a control scheme to manage abnormal situations. Compared to network reinforcement, the control of Dispersed Generation Unit (DGU) productions is attractive, in so far the violations take place only a fraction of the time [2]. The choice of an appropriate scheme is also dictated by the information exchange between the DSO and the other actors in the system.

Corrective control can be realized according to different architectures: centralized [3], [4], [5], [6], decentralized [7], distributed [5], and hierarchical [8]. Furthermore, voltage and thermal violations can be corrected separately [3], [5], [6] or

Work supported by Public Service of Wallonia, Dept. of Energy and Sustainable Building, within the framework of the GREDOR project. The authors are with the Dept. of Electrical Engineering and Computer Science, University of Liège, Belgium. T. Van Cutsem is with the Funds for Scientific Research (FNRS) at the same department. jointly [4], [7], [8]. Using an Optimal Power Flow (OPF), Ref. [5] discussed the impact of centralized and distributed voltage control schemes on potential penetration of dispersed generation, while in [6], using a similar technique, thermal constraints are primarily managed in a centralized manner, with voltage constraints also included in the formulation. In [7] a decentralized approach was proposed for the real-time control of both constraints, using voltage and apparent power flow sensitivities to identify the most effective control actions. A hierarchical model based controller was proposed in [8] were the upper level coordinates different types of control devices relative to a set of prioritized objective functions. The work in [9] proposes a composable method that uses real-time power setpoints in order to control the grid in a scalable and reliable way. The approach enables the behaviour of a complex electrical system to emerge as a property of a combination of interacting agents.

However, relatively few references deal with an automatic, closed-loop control to smoothly steer the system and bring it back within the security limits, while compensating for model inaccuracies. Model Predictive Control (MPC) offers such capabilities [10], [11]. Reference [3] proposed a centralized voltage control scheme inspired by MPC. The problem was formulated as a receding-horizon multi-step optimization using a simple sensitivity model. This formulation was further extended in [4] to jointly manage voltage and thermal constraints.

This paper proposes a centralized, joint voltage and thermal control scheme, relying on appropriate measurement and communication infrastructures. DGUs are categorized as dispatchable and non-dispatchable, respectively, and three contexts of application are presented according to the nature of the DGUs and the aforementioned information exchanges. This extends the work in [3], [4] by considering a new objective function. Namely, the deviations with respect to power schedules are minimized for the dispatchable DGUs, while the others can operate as much as possible according to the maximum power tracking strategy. The formulation is such that the correction sent to DGUs vanishes as soon the operating constraints are no longer binding.

The paper is organized as follows. Section II details the multi-step constrained optimization, while Section III discusses various contexts of application. Simulation results are reported in Section IV, and concluding remarks in Section V. 


\section{CONSTRAined MUlTi-STEP OPTIMIZATION}

\section{A. Principle}

The proposed control scheme is inspired of the principle of MPC [10], [11]. One important feature of MPC is the closedloop nature of the controller, which enables compensating for inaccurate prediction of system response due to the model simplification, noisy measurements, actuator failures, and other uncertainties.

A multi-step receding-horizon optimization is thus considered which predicts the system evolution at the future $N_{p}$ discrete time steps. Given the nature of the problem (violation alleviation within a few minutes), a sensitivity model is appropriate, while little demanding in terms of data [3], [4]. At a given discrete time $k$, an optimal sequence of control actions $\boldsymbol{u}(k+i)\left(i=0, \ldots, N_{c}-1\right)$ is determined for the $N_{c}$ future time steps, with the objective of bringing the system within limits by the end of the prediction horizon. According to the MPC principle, only the first component $\boldsymbol{u}(k)$ of the sequence is applied, at time $k$. At the next time step, based on new received measurements, the whole procedure is repeated.

\section{B. Formulation}

The control variables are the active $\left(\boldsymbol{P}_{g}\right)$ and reactive $\left(\boldsymbol{Q}_{g}\right)$ powers produced by the DGUs:

$$
\boldsymbol{u}(k)=\left[\begin{array}{ll}
\boldsymbol{P}_{g}^{T}(k) & \boldsymbol{Q}_{g}^{T}(k)
\end{array}\right]^{T}
$$

where ${ }^{T}$ denotes transposition. These variables have timevarying reference values, denoted by $\boldsymbol{P}_{r e f}(k)$ and $\boldsymbol{Q}_{r e f}(k)$, respectively, whose choice will be discussed in Section III.

The objective is to minimize the sum of squared deviations, over the $N_{c}$ future steps, between the controls and their references:

$$
\begin{aligned}
\min _{\boldsymbol{P}_{g}, \boldsymbol{Q}_{g}, \boldsymbol{\varepsilon}} & \sum_{i=0}^{N_{c}-1}\left\|\boldsymbol{P}_{g}(k+i)-\boldsymbol{P}_{r e f}(k+i)\right\|_{\boldsymbol{R}_{1}}^{2}+ \\
& +\sum_{i=0}^{N_{c}-1}\left\|\boldsymbol{Q}_{g}(k+i)-\boldsymbol{Q}_{r e f}(k+i)\right\|_{\boldsymbol{R}_{2}}^{2}+\|\boldsymbol{\varepsilon}\|_{\boldsymbol{S}}^{2}
\end{aligned}
$$

where the diagonal weighting matrices $\boldsymbol{R}_{1}$ and $\boldsymbol{R}_{2}$ allow prioritizing the controls, with lower values assigned to reactive than to active power deviations. The last term in (2) involves the slack variables $\varepsilon$ aimed at relaxing the inequality constraints in case of infeasibility; the entries of the diagonal matrix $\boldsymbol{S}$ are given very high values. The above objective is minimized subject to the linearized system evolution:

$$
\begin{aligned}
& \text { for } i=1, \ldots, N_{p} \text { : } \\
& \boldsymbol{V}(k+i \mid k)=\boldsymbol{V}(k+i-1 \mid k)+ \\
& +\boldsymbol{S}_{V}[\boldsymbol{u}(k+i-1)-\boldsymbol{u}(k+i-2)] \\
& \boldsymbol{I}(k+i \mid k)=\boldsymbol{I}(k+i-1 \mid k)+ \\
& +\boldsymbol{S}_{I}[\boldsymbol{u}(k+i-1)-\boldsymbol{u}(k+i-2)]
\end{aligned}
$$

where $\boldsymbol{V}(k+i \mid k)$ and $\boldsymbol{I}(k+i \mid k)$ are the predicted bus voltages and branch currents, and $\boldsymbol{S}_{V}$ and $\boldsymbol{S}_{I}$ are sensitivity matrices of those variables with control changes. The use of this static model is justified by the fast response of powerelectronics based DGUs. The prediction is initialized with $\boldsymbol{V}(k \mid k)$ and $\boldsymbol{I}(k \mid k)$ set to the last received measurements. Finally, the following inequality constraints are imposed:

for $i=1, \ldots, N_{p}$ :

$$
\begin{aligned}
& -\varepsilon_{1} \mathbf{1}+\boldsymbol{V}^{\text {low }}(k+i) \leq \boldsymbol{V}(k+i \mid k) \\
& \boldsymbol{V}(k+i \mid k) \leq \boldsymbol{V}^{\text {up }}(k+i)+\varepsilon_{2} \mathbf{1} \\
& \boldsymbol{I}(k+i \mid k) \leq \boldsymbol{I}^{\text {up }}(k+i)+\varepsilon_{3} \mathbf{1}
\end{aligned}
$$

and for $i=0, \ldots, N_{c}-1$ :

$$
\begin{aligned}
& \boldsymbol{u}^{\text {min }} \leq \boldsymbol{u}(k+i \mid k) \leq \boldsymbol{u}^{\max } \\
& \Delta \boldsymbol{u}^{\text {min }} \leq \boldsymbol{u}(k+i \mid k)-\boldsymbol{u}(k+i-1 \mid k) \leq \Delta \boldsymbol{u}^{\max }
\end{aligned}
$$

where $\boldsymbol{u}^{\min }, \boldsymbol{u}^{\max }, \Delta \boldsymbol{u}^{\min }$ and $\Delta \boldsymbol{u}^{\max }$ are the lower and upper limits on DGU outputs and on their rate of change. $\varepsilon_{1}$, $\varepsilon_{2}$ and $\varepsilon_{3}$ are the components of $\varepsilon$, and $\mathbf{1}$ denotes a unit vector. Eq. (8) includes the limits on reactive powers of DGUs, which are updated at each time step based on the measured active power and terminal voltage.

\section{Sensitivity matrices}

The sensitivity matrix $\boldsymbol{S}_{V}$ can be obtained off-line from the transposed inverse of the power flow Jacobian matrix. As an alternative, each column can be computed by running a power flow calculation with one DGU power slightly modified, and simply dividing the variations of the monitored bus voltages by that power variation. This matrix can be updated infrequently, the errors being compensated by the MPC scheme [3].

The sensitivity matrix $\boldsymbol{S}_{I}$ should be updated more frequently, due to the higher variability of currents. The sensitivity of the branch current $I_{j}$ with respect to $i$-th DGU active (resp. reactive) power $P_{g i}$ (resp. $Q_{g i}$ ) can be obtained as [4]:

$$
\begin{gathered}
\frac{\partial I_{j}}{\partial P_{g i}} \approx \frac{1}{V_{k}} \frac{P_{j}}{S_{j}} \frac{\partial P_{j}}{\partial P_{g i}} \approx \frac{P_{j}}{S_{j}} \\
\frac{\partial I_{j}}{\partial Q_{g i}} \approx \frac{1}{V_{k}} \frac{Q_{j}}{S_{j}} \frac{\partial Q_{j}}{\partial Q_{g i}} \approx \frac{Q_{j}}{S_{j}}
\end{gathered}
$$

where $P_{j}, Q_{j}$ and $S_{j}$ are respectively the active, reactive and apparent power flows in the branch, and $V_{k}$ the voltage at the bus where the current is measured. The voltage is simply taken equal to $1 \mathrm{pu}$. The above approximations assume that $P_{j}$ (resp. $Q_{j}$ ) does not change much when $Q_{g i}$ (resp. $P_{g i}$ ) is varied, and the change of $P_{j}$ (resp. $Q_{j}$ ) is equal to the change in $P_{g i}$ (resp. $Q_{g i}$ ). Note that this approximation applies only if the $j$-th branch is on the path from the $i$-th DGU to the HV/MV tranformer; otherwise, a zero sensitivity is assumed since the branch current would not change with the DGU power change. Note also that using $(10,11)$ requires to have the branch equipped with active and reactive power flow measurements.

In our tests $\boldsymbol{S}_{I}$ has been updated at each discrete step while $\boldsymbol{S}_{V}$ has been kept constant at all operating points. The low impact on control actions of the latter simplification are illustrated in Appendix I. 


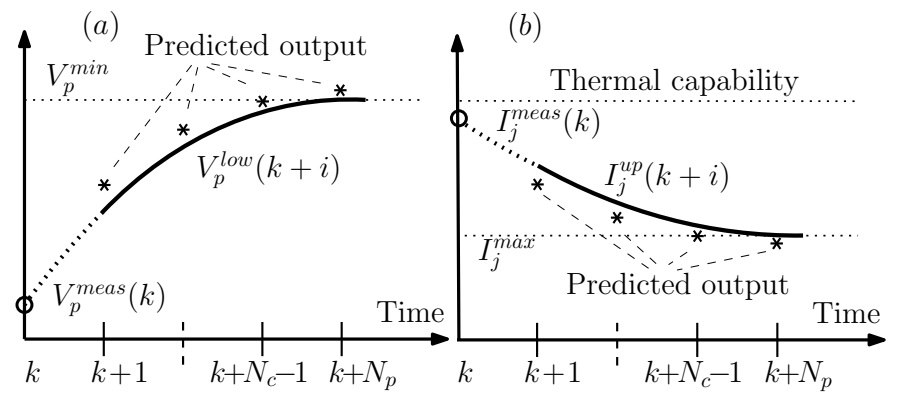

Figure 1. Progressive tightening of voltage and current bounds

\section{Bound tightening}

To obtain a smooth system evolution, the bounds $\boldsymbol{V}^{\text {low }}(k+i), \boldsymbol{V}^{u p}(k+i)$ and $\boldsymbol{I}^{u p}(k+i)$ on the predicted voltages and currents are tightened progressively [10]. An exponential evolution with time has been considered, as shown in Fig. 1 for respectively a lower voltage (Fig. 1.(a)) and a current limit (Fig. 1.(b)). The circles indicate voltage or current values measured at time $k$, which fall outside the acceptable range. The limits imposed at the successive times $k+1, \ldots, k+N_{p}$ are shown with solid lines. They force the voltage or current of concern to enter the acceptable range at the end of the prediction horizon. Taking the lower voltage limit as an example, the variation is given by $\left(i=1, \ldots, N_{p}\right)$ :

$$
V_{p}^{\text {low }}(k+i)=V_{p}^{\text {min }}-\left(V_{p}^{\text {min }}-V_{p}^{\text {meas }}(k)\right) e^{-i / T_{s}}
$$

where $p$ is the bus of concern and $V_{p}^{\text {meas }}(k)$ is the measurement received at time $k$. If it does not exceed the desired limit, i.e. if $V_{p}^{\text {meas }}(k) \geq V_{p}^{\text {min }}$, the latter is used as constant bound in (5), at all future times, i.e. $V_{p}^{\text {low }}(k+i)=V_{p}^{\text {min }}, \quad i=$ $1, \ldots, N_{p}$. The time constant $T_{s}$ is chosen to have the last predicted output inside the acceptable limits.

Similar variations are considered for the upper voltage and the current limits. As regards the latter, the $I^{\max }$ value is set conservatively below the effective thermal capability monitored by the corresponding protection, as shown in Fig. 1.(b).

\section{E. Response time of the controller}

The objective of the proposed control is to correct limit violations in real-time. As will be shown in the Results, the typical sampling period $\left(t_{k+1}-t_{k}\right)$ is in the order of 10 seconds, and the number of future steps is $N_{c}=3$. Thus, the control horizon is $3 \times 10=30$ seconds. which is fast enough for emergency control purposes.

Note that this is an average value but, depending on the situation (in particular the number of active inequality constraints) the violations are cleared somewhat faster or slower.

\section{CONTEXTS OF APPLICATION AND CHOICE OF REFERENCE VALUES}

The above MPC formulation can accommodate various contexts of application, depending on the interactions and information transfers between entities acting on the DGUs, in accordance with the regulatory policy. This leads to defining a number of operating modes, which are depicted in Fig. 2.
The proposed controller is executed by a central entity, typically the DSO, which collects real-time measurements. The latter consists of active and reactive productions and terminal voltages of the DGUs, active and reactive power flows in critical (potentially congested) branches, and some other bus voltages. Thus, the controller relies on a dedicated measurement and communication infrastructure but, as suggested in Fig. 2, it could also rely on the results of a state estimator, for improved system monitoring.

Once the controller observes (or predicts) limit violations, it computes and sends active and/or reactive power corrections to the DGUs of concern. The latter are the differences between the reference and the computed controls, i.e.

$$
\begin{aligned}
\Delta \boldsymbol{P}_{c o r}(k) & =\boldsymbol{P}_{r e f}(k)-\boldsymbol{P}_{g}(k) \\
\Delta \boldsymbol{Q}_{\text {cor }}(k) & =\boldsymbol{Q}_{\text {ref }}(k)-\boldsymbol{Q}_{g}(k)
\end{aligned}
$$

Note that these corrections stay at zero as long as no limit violation is observed (or predicted), and come back to zero (together with the objective function (2)) as soon as operation is no longer constrained, as explained in the sequel.

Furthermore, a distinction is made between dispatchable and non-dispatchable DGUs. The latter are typically wind turbine or photovoltaic units operated for Maximum Power Point Tracking (MPPT). In the absence of operating constraints, they are left to produce as much as it can be obtained from the renewable energy source. The dispatchable units, on the other hand, have their productions modulated, according to market opportunities or balancing needs, for instance.

\section{A. Mode 1}

This mode applies to non-dispatchable units. For MPPT purposes, at each time step $k$, the reference $P_{\text {ref } i}(k)$ of the $i$ th DGU should be set to the maximum power available on that unit. This information is likely to be available to the DGU MPPT controller, but is seldom transmitted outside. An alternative is to estimate that power from the measurements $\boldsymbol{P}_{\text {meas }}$. Considering the short control horizon of concern here, a simple prediction is given by the "persistence" model:

$$
\boldsymbol{P}_{\text {ref }}(k+i)=\boldsymbol{P}_{\text {meas }}(k)+\Delta \boldsymbol{P}_{\text {cor }}(k-1), \quad i=0, \ldots, N_{c}
$$

As long as no power correction is applied, the last term is zero and $\boldsymbol{P}_{\text {meas }}$ is used as a short-term prediction of the available power. When a correction is applied, the right-hand side in (15) keeps track of what was the available power before a correction started being applied. Using this value as reference allows resetting the DGUs under the desired MPPT mode as soon as system conditions improve.

These features are further illustrated through a hypothetical scenario in Appendix II.

More accurate prediction can be used, if data are available. That would result in the right-hand side of (15) varying with time $k+i$. In this work, however, only the persistence model was considered. 

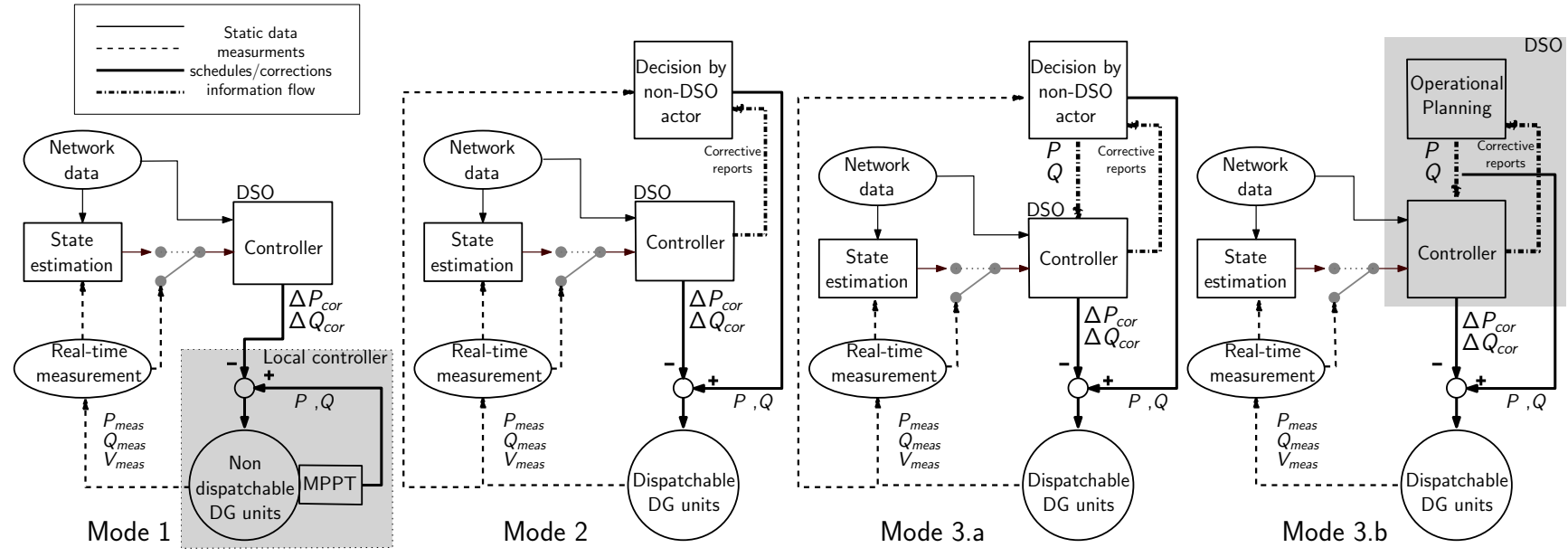

Figure 2. Contexts of application of the proposed control scheme

\section{B. Mode 2}

This mode applies to DGUs that are dispatchable but under the control of another actor than the DSO. Thus, the latter does not know the power schedule of the units of concern. In order to avoid interference with that non-DSO actor, the last measured power productions are taken as reference values over the next $N_{c}$ time steps:

$$
\boldsymbol{P}_{\text {ref }}(k+i)=\boldsymbol{P}_{\text {meas }}(k), \quad i=0, \ldots, N_{c}
$$

On the other hand, if a control action has been applied by the DSO, to preserve network security, this action should not be counteracted by a subsequent non-DSO action in order to avoid conflicts, leading for instance to oscillations. In other words, the DSO is assumed to "have the last word" in terms of corrective actions, since it is the entity responsible for network security.

In this mode, the controller lacks information to anticipate the DGU power evolution. Hence, the corrections $(13,14)$ will be applied ex post, after the measurements have revealed the violation of a (voltage or current) constraint.

\section{Mode 3}

This mode relates to dispatchable DGUs whose power schedules are known by the controller, either because this information is transmitted by the non-DSO actors controlling the DGUs (see variant 3.a in Fig. 2) or because the DSO is entitled to directly control the DGUs (see variant 3.b in Fig. 2). The latter case may also correspond to schedules determined by the DSO operational planning. Unlike in Mode 2, the schedule imposed to the units is known by the controller, which can anticipate a possible violation under the effect of the scheduled change, and correct the productions ex ante. Although different from a regulatory viewpoint, Modes 3.a and 3.b are treated in the same way.

Figure 3 shows how the $N_{c}$ future $\boldsymbol{P}_{\text {ref }}$ values are updated with the known schedule before being used in the objective (2). As long as the schedule does not change within the $N_{c}$ future time steps (see Fig. 3.a), $\boldsymbol{P}_{\text {ref }}$ remains unchanged; otherwise, the interpolated values are used.

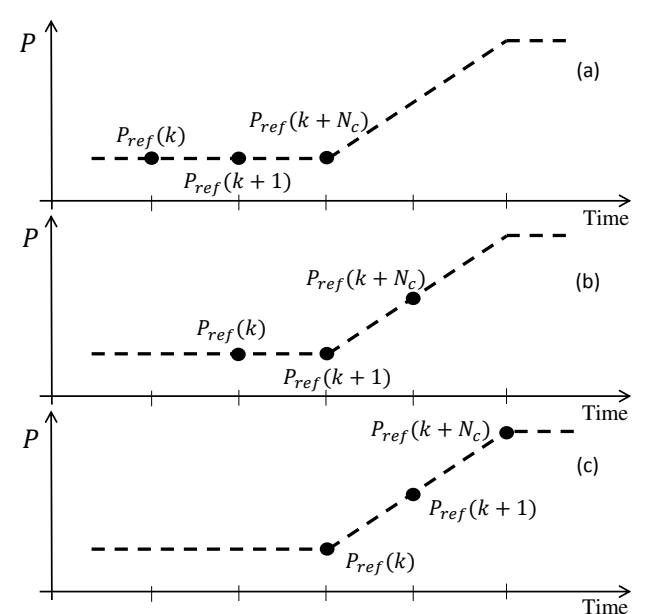

Figure 3. Mode 3: updating the $P_{\text {ref }}$ values over three successive times

\section{Remarks}

1) In principle the aforementioned choices also apply to the reference reactive powers $\boldsymbol{Q}_{r e f}$. However, it is quite common to operate DGUs at unity power factor, to minimize internal losses, which amounts to setting $\boldsymbol{Q}_{r e f}$ to zero, and corresponds to Mode 3.

2) To make system operation smoother and more secure, the identified limit violations and the corresponding corrections applied by the controller to DGUs should be communicated back to the non-DSO actors or the operational planners, as suggested by the dash-dotted arrows in Fig. 2.

\section{Simulation REsults}

\section{A. Test system and simulation tools}

In this section the performance of the proposed controller, in each of the aforementioned modes, is illustrated on the 75-bus, $11-\mathrm{kV}$ distribution network whose one-line diagram is shown in Fig.4. It is connected to the upper voltage level (referred to as external grid) through a $33 / 11 \mathrm{kV}$ transformer equipped with load tap changer. 


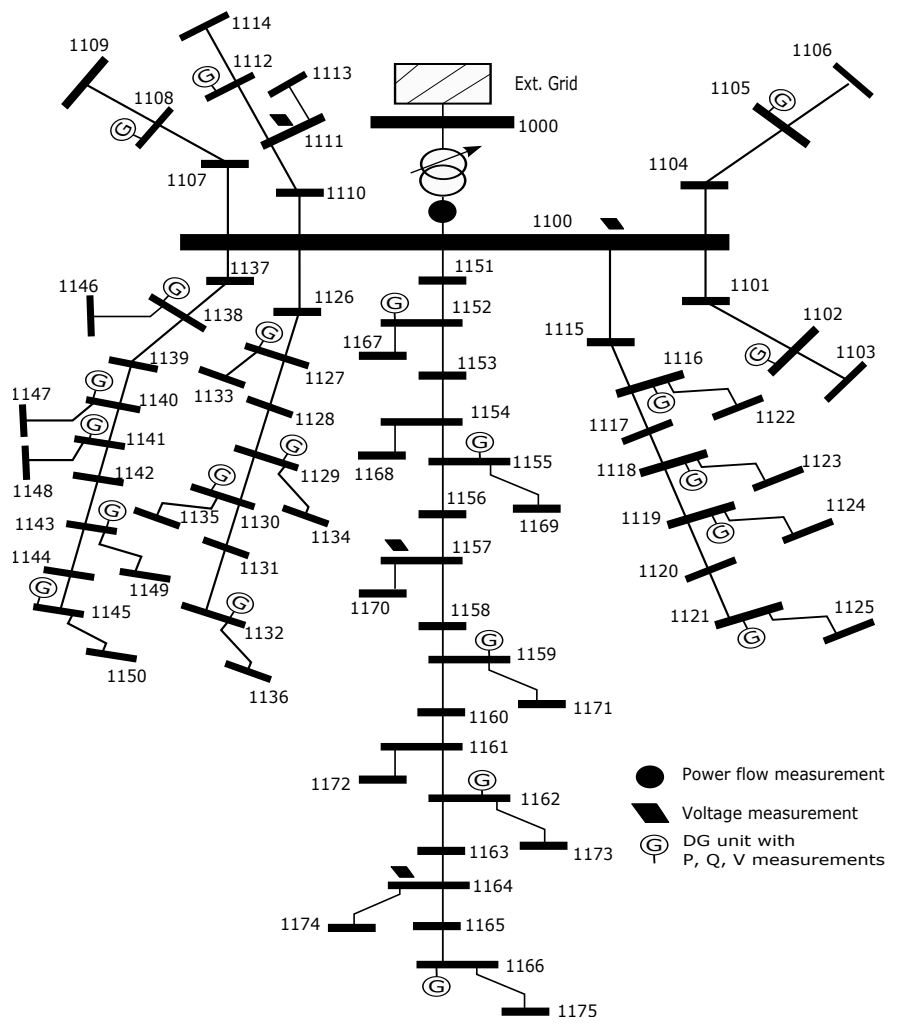

Figure 4. Network one-line diagram

The network hosts 22 DGUs, consisting of 3.33-MVA doubly fed induction generators driven by 3-MW wind-turbines, and 3-MVA synchronous generators driven by $2.55-\mathrm{MW}$ turbines, respectively. The DGU models and parameters were taken from [12], [13], and simplified in accordance with the type of dynamics considered in this work.

The network feeds 52 loads, of which 38 are modelled as constant current for active power and constant impedance for reactive power, and 14 with induction motors.

The measurements are received by the controller every $10 \mathrm{~s}$. It is assumed that the terminal voltage and the active/reactive power outputs of the 22 DGUs are measured, as well as the active and reactive power flows in the transformer and the voltage on its $11-\mathrm{kV}$ side. The controller sends corrections every $10 \mathrm{~s}$. The DGUs respond to them with time constants in the order of a few seconds, due to their internal dynamics. The prediction and control horizons are set to $N_{p}=N_{c}=3$. This yields a good compromise between a sufficient number of MPC steps and a short enough response time to correct violations [3], [4]. The matrices $\boldsymbol{R}_{1}, \boldsymbol{R}_{2}$ and $\boldsymbol{S}$ in (2) are diagonal with entries equal to 1 for reactive powers, 25 for active powers, 500 for the slack variables $\varepsilon_{1}$ and $\varepsilon_{2}$ (see $(5,6)$ ) and 5000 for $\varepsilon_{3}$ (see (7)). This relative weighting is an easy way to give the desired priorities to the various variables and are likely to be suitable for many systems.

It must be emphasized that the changes in operating point applied to the system, such as wind variations, load increases and scheduled changes, have been made faster than in reality for a legible presentation of the results.

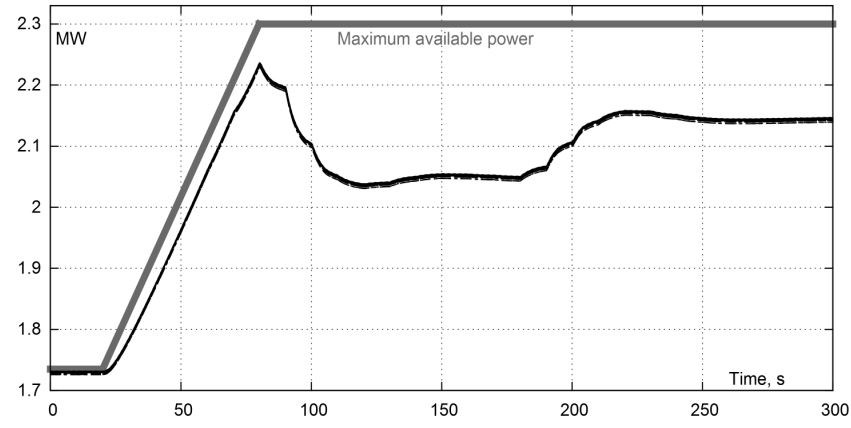

Figure 5. Scenario A: Active power produced by DGUs

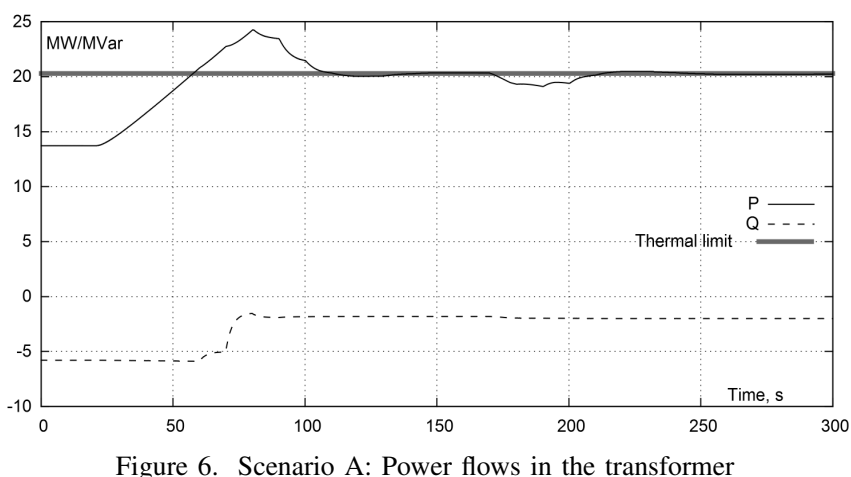

The simulations were carried out with RAMSES, a detailed time simulation program developed at the University of Liège [14]. The proposed discrete-time controller is solving the Quadratic Programming problem (2-9) with the VE17AD library from Harwell [15].

\section{B. Scenario A: Mode 1}

All 22 DGUs are assumed to be driven by wind turbines, operated for MPPT. Thus the control of all DGUs is in Mode 1. Initially the dispersed generation exceeds the load, and the distribution network is injecting active power into the external grid. At the same time, the DGUs are operating at unity power factor, and the distribution network draws reactive power from the external grid.

A $10 \%$ increase in wind speed takes place from $t=20$ to $t=80 \mathrm{~s}$, as shown in Fig. 5. This results in an increase of the active power flow in the transformer, as shown in Fig. 6. At $t=60 \mathrm{~s}$, the thermal limit of the latter, shown with heavy line in Fig. 6, is exceeded. This is detected by the controller through a violation of the constraint (7) at $t=70 \mathrm{~s}$.

The controller corrects this congestion problem by acting first on the DGU reactive powers, which have higher priority through the weighting factors. Figure 7 shows that the controller makes some DGUs produce reactive powers, to decrease the import (and, hence, the current) through the transformer. The latter effect can be seen from Fig. 6. However, the sole correction of DGU reactive powers cannot alleviate the overload, and from $t=80 \mathrm{~s}$ on, the controller curtails the active power of wind turbines, as shown by Fig. 5. The overload is fully corrected at $t=120 \mathrm{~s}$.

To illustrate the ability of the proposed control to steer the DGUs back to MPPT, the system operating conditions 


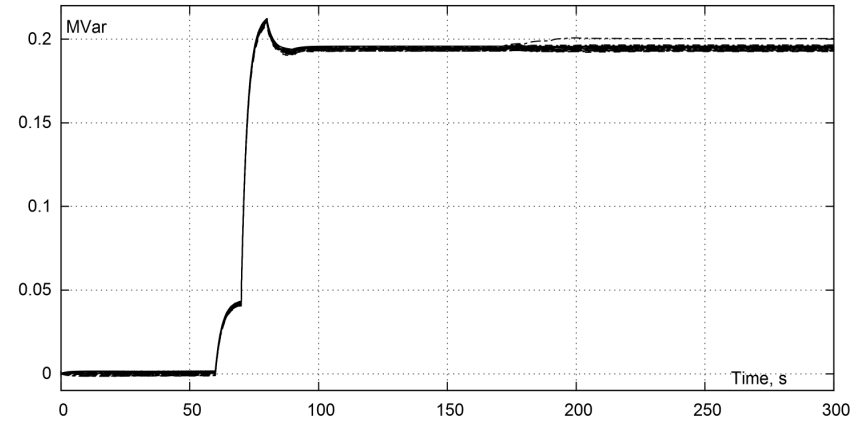

Figure 7. Scenario A: Reactive power produced by DGUs

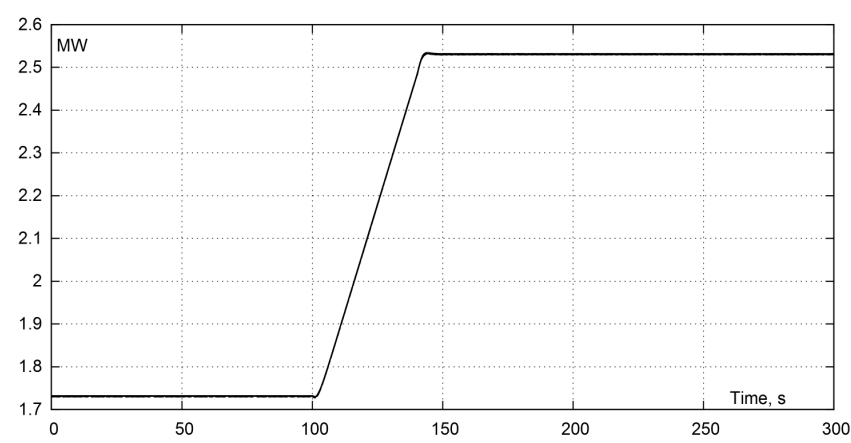

Figure 8. Scenario B: Active power produced by dispatchable units

are relieved by simulating a 4.1-MW load increase starting at $t=170 \mathrm{~s}$. The corresponding decrease of the active power flow in the transformer can be observed in Fig. 6. This leaves some space to restore part of the curtailed DGU active powers. As expected, the controller increases the DGU productions until the transformer current again reaches its limit, at around $t=210 \mathrm{~s}$. Figure 5 shows that, indeed, the active productions get closer to the maximum power available from wind.

The unpredicted thermal limit violation caused by the initial wind increase was corrected ex post, as explained in Section III.A. It is interesting to note that, on the contrary, when taking advantage of the load relief, the controller steers the system in such a way that it does not exceed the thermal limit.

On a standard laptop, the elapsed time to solve the optimizations in this scenario, is varying from zero up to 32 milliseconds, depending on the computational efforts at each time step.

\section{Scenario B: Modes 1 and 2 combined}

It is now assumed that nine units, connected to buses 1118 , $1119,1129,1132,1138,1141,1155,1159$, and 1162 (see Fig. 4), are driven by wind turbines and are non dispatchable. They are thus operated in Mode 1 . However, since the wind speed is assumed constant in this scenario, the productions of those units remain constant.

The remaining 13 DGUs use synchronous generators and are dispatchable. They are operated in Mode 2. An increase of their active power by an actor other than the DSO, thus not known by the controller, takes place from $t=100$ to $t=140 \mathrm{~s}$, as shown in Fig. 8. The schedule leaves the reactive powers unchanged. Since the initial network voltages are close
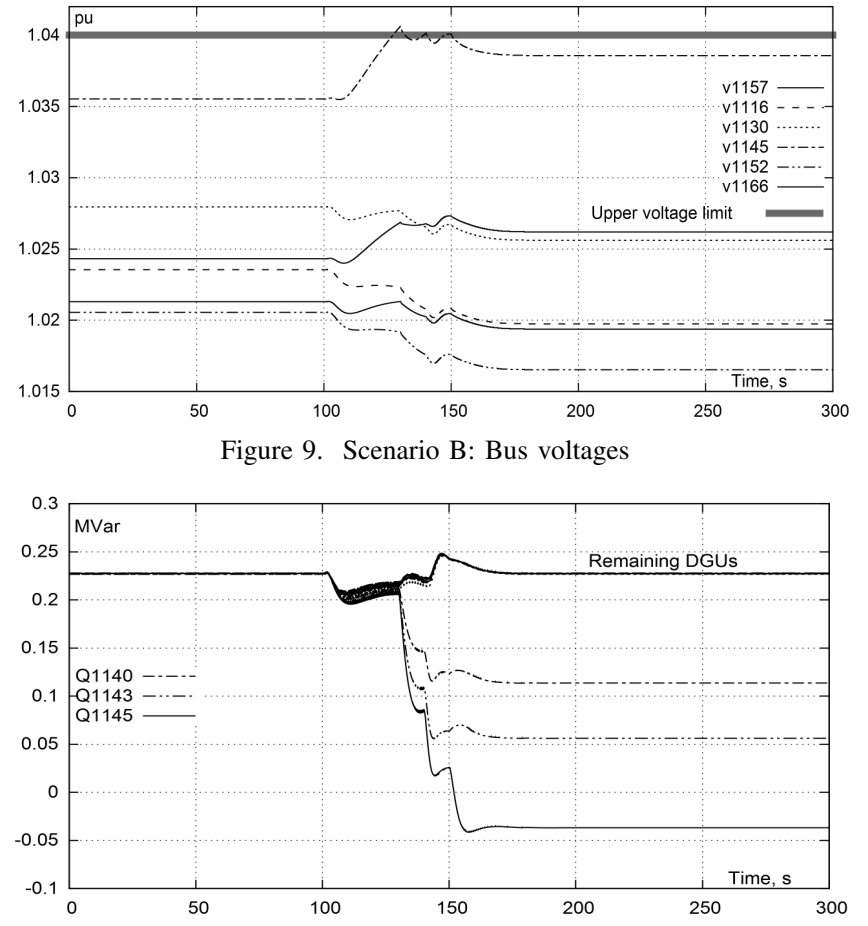

Figure 10. Scenario B: Reactive power produced by dispatchable units

to the admissible upper limit, shown with heavy line in Fig. 9, the system undergoes high voltage problems.

The controller does not send corrections until $t=130 \mathrm{~s}$, when the voltage at bus 1145 exceeds the limit. Over the 40 seconds that follow this limit violation, the controller adjusts the reactive powers of both dispatchable and nondispatchable units, as shown by Figs. 10 and 11. It is easily seen that different corrections are applied to different DGUs, depending on their locations in the system. It is also seen from Fig. 9 that the voltage at bus 1145 crosses the limit several times, followed by reactive power adjustments. These ex post corrections were to be expected since, in this example, the DGUs are either in Mode 1 or in Mode 2.

\section{Scenario $C$ : Modes 1 and 3 combined}

In this last scenario, some DGUs are non dispatchable and operated in Mode 1, while the dispatchable ones are operated in Mode 3, with their schedules known by the controller. The latter may come, for instance, from operational planning decisions.

Two successive changes of DGU active powers are considered: (i) an unforeseen wind speed change from $t=20$ to $t=70 \mathrm{~s}$ increasing the production of the non-dispatchable units, and (ii) a power increase of the dispatchable units scheduled to take place from $t=150$ to $t=190 \mathrm{~s}$. The corresponding active power generations are shown in Fig. 12.

Figure 13 shows the resulting evolution of a few bus voltages. The increase in wind power makes them approach their limit, shown with heavy line. Without a corrective action, the subsequent scheduled change would cause a limit violation. However, as explained in Section III.C, the latter change is 


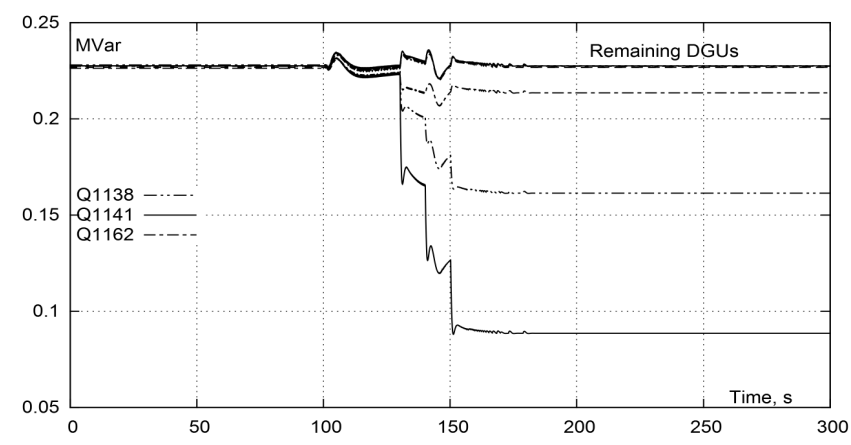

Figure 11. Scenario B: Reactive power produced by non-dispatchable units

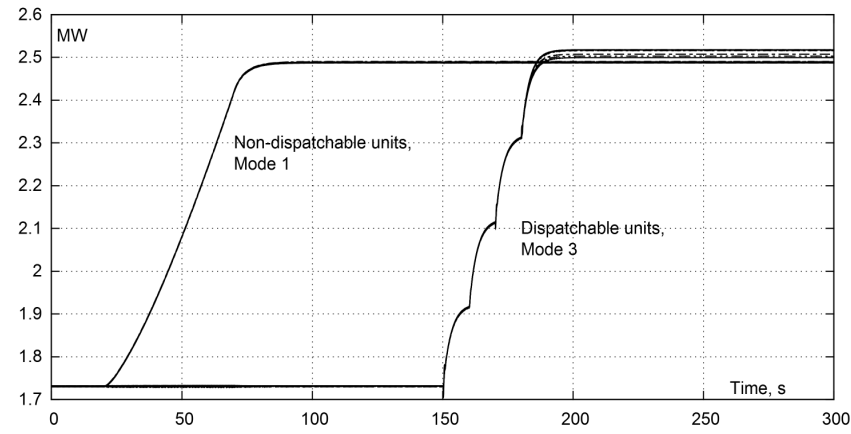

Figure 12. Scenario C: Active power produced by various units

anticipated by the controller, through the $P_{\text {ref }}$ values updated as shown in Fig. 3. Therefore, the controller anticipatively adjusts the DGU reactive powers, as can been in Figs. 14 and 15 , with the result that no voltage exceeds the limit, while all the active power changes are accommodated. The controller anticipative reaction is clearly seen in Fig. 13, where the voltage decrease resulting from the reactive power adjustment counteracts the increase that accompanies the active power increase, leading the highest voltage to land on the upper limit.

\section{CONCLUSION}

In this paper, a real-time, centralized, receding-horizon control scheme has been proposed, aimed at correcting unacceptable voltages and thermal overloads. Based on the principle of MPC, it relies on a multi-step constrained optimization. The objective is to keep the DGU productions as close as possible to their reference evolutions, while alleviating voltage and thermal problems when they occur. The quadratic objective and the progressive enforcement of operating constraints yield a smooth, while effective control of the DGUs.

Three modes of operation have been presented in some detail, allowing to control both dispatchable and nondispatchable units, to let the latter operate at MPPT whenever possible, and to deviate the former as few as possible from the desired schedules. Through weighting factors, priority is given to reactive power over active power corrections. Depending on the information available to the controller, it corrects the violations either anticipatively or after they have taken place.

The various applications have been illustrated through the successful results of dynamic simulations involving a test system with 22 DGUs, which led to a coordinated control problem of significant complexity.
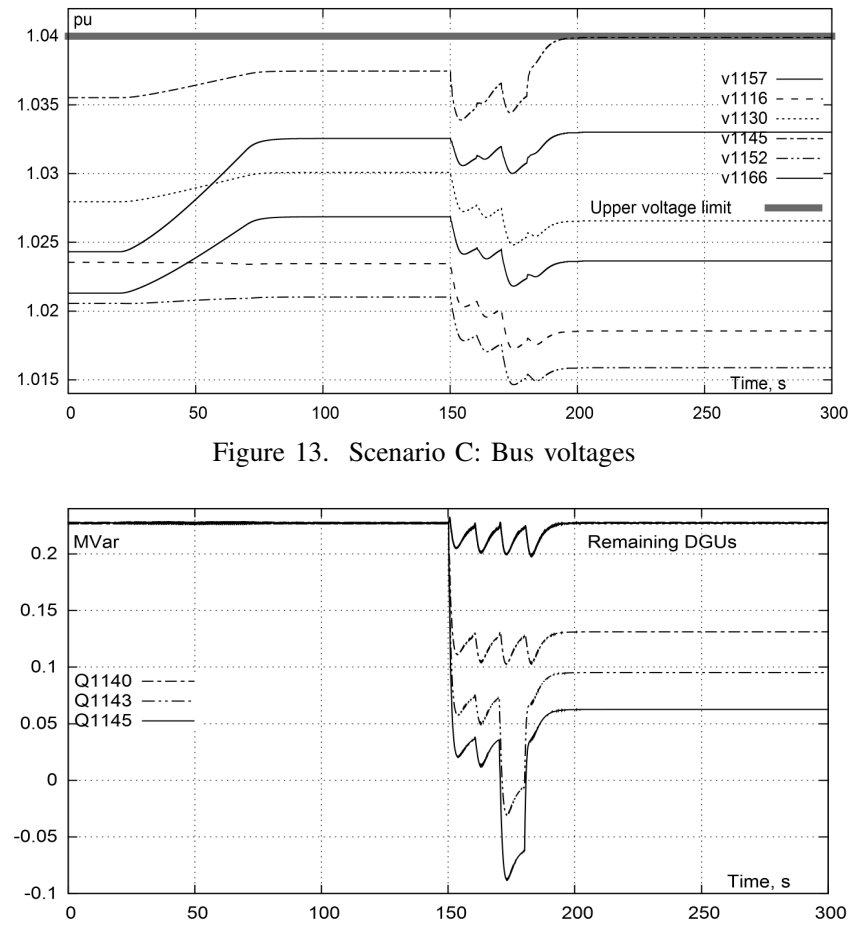

Figure 14. Scenario C: Reactive power produced by dispatchable units

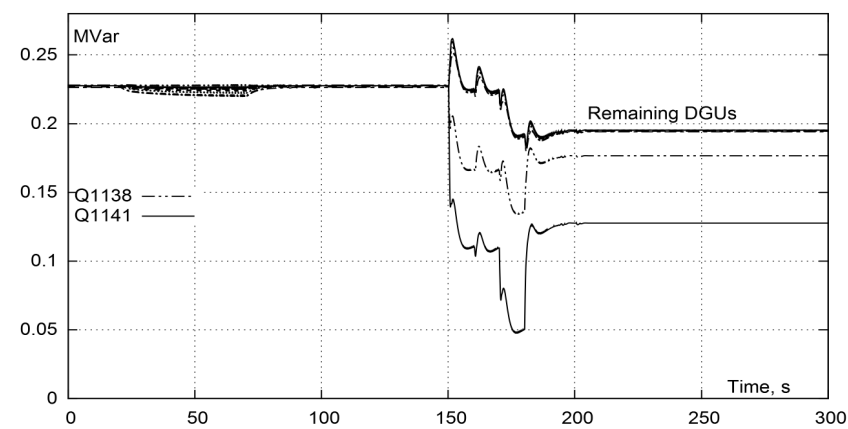

Figure 15. Scenario C: Reactive power produced by non-dispatchable units

One of the current extension of the method deals with the prediction of short-term system evolution which can lead to avoid some violations. Moreover, besides real-time measurements, the DSO controller can take advantage of a state estimator for better observation of the system behaviour.

\section{APPENDIX I. CONTROLLER ROBUSTNESS EVALUATION}

The choice of keeping $\boldsymbol{S}_{V}$ constant at all operating points leads to less computational burden. On the other hand, the impact on the controller response should be evaluated. A simple example of such a validation is presented in Fig. 16. The controller addresses an over-voltage situation, caused by increasing active power generations. Two sensitivity matrices were calculated at two significantly different operating points. Namely, accurate sensitivities were computed at the initial operating point of the system, while inexact values were computed around an operating point with all productions of DGUs set to zero. It is worth mentioning that, in this test, the 


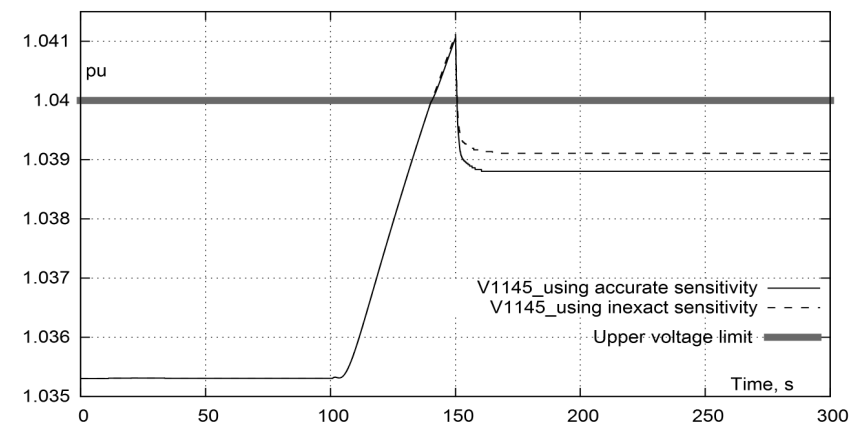

Figure 16. Voltage evolution at bus 1145 with accurate and approximate sensitivities

average deviation of the sensitivity matrix elements is around $10 \%$ with respect to their accurate values.

As it can be seen in Fig. 16, although the controller relies on an imprecise sensitivity model, the violation is completely alleviated and the voltage evolution (dashed line) is remarkably close to the one obtained with accurate sensitivities (solid line).

\section{APPENDiX II. Simple ILlustrative EXAMPlE OF Mode 1}

The following hypothetical example illustrates the controller performance in Mode 1. The example encompasses three successive events. It is detailed in Table I, where the numbers refer to a single DGU and are percentages of its rated power.

At time $k$, the DGU is in MPPT mode and the operating conditions are normal. Thus, $P_{\text {ref }}$ is set to the received power measurement $P_{\text {meas }}=90$. All constraints being satisfied, the optimization leaves the production unchanged, i.e. $P_{g}=P_{r e f}$ and $\Delta P_{\text {cor }}=0$. At time $k+1$ an emergency is detected in the network (in the form of a branch overload or an abnormal voltage). The optimization yields $P_{g}=80\left(\Delta P_{\text {cor }}=10\right)$ at time $k+1$ and $P_{g}=75\left(\Delta P_{\text {cor }}=15\right)$ at time $k+2$. The effect of each correction is seen on the measured value received at the next time. To keep the example short, it is assumed that the emergency is cleared in two steps: the network is back to normal at time $k+3$. Note that $P_{r e f}$, obtained from (15), keeps track of the maximum power that the DGU could produce, if it was not curtailed.

At time $j>k+3$ an increase in wind power (detected by the increase of $P_{\text {meas }}$ from 75 to 82) causes a return to emergency, corrected at time $j+1$ by increasing the curtailment by the same amount. Note that $P_{\text {ref }}$ has been updated accordingly to the new maximum power that the DGU could produce which has increased while the emergency was being corrected by the controller.

At time $\ell>j+1$ the cause of the initial emergency has been cleared, and it is found that $P_{g}$ can be restored to $P_{r e f}$ without creating an emergency. The controller adjusts $\Delta P_{\text {cor }}$ in two steps, from 22 to 12 , and from 12 to zero. Consequently, the DGU recovers its MPPT operation, as confirmed by the value of $P_{\text {meas }}$ received at time $\ell+2$.
TABLE I. ILluStrative EXAMPLE OF OPERATION IN MODE 1

\begin{tabular}{c||c|c|c|c||c|c}
\hline Time & $P_{\text {meas }}$ & $P_{\text {ref }}$ & $P_{g}$ & $\Delta P_{\text {cor }}$ & network & DGU \\
\hline \hline$k$ & 90 & 90 & 90 & 0 & normal & full power \\
\hline$k+1$ & 90 & 90 & 80 & 10 & emerg. & full power \\
\hline$k+2$ & 80 & $80+10=90$ & 75 & 15 & emerg. & curtailed \\
\hline$k+3$ & 75 & $75+15=90$ & 75 & 15 & normal & curtailed \\
\hline \hline$j$ & 82 & $82+15=97$ & 75 & 22 & emerg. & curtailed \\
\hline$j+1$ & 75 & $75+22=97$ & 75 & 22 & normal & curtailed \\
\hline \hline$\ell$ & 75 & $75+22=97$ & 85 & 12 & normal & curtailed \\
\hline$\ell+1$ & 85 & $85+12=97$ & 97 & 0 & normal & restoring to MPPT \\
\hline$\ell+2$ & 97 & 97 & 97 & 0 & normal & full power \\
\hline
\end{tabular}

\section{REFERENCES}

[1] Q. Gemine, E. Karangelos, D. Ernst and B. Cornelusse, Active network management: planning under uncertainty for exploiting load modulation, IREP Symposium-Bulk Power System Dynamics and Control -IX, Rethymnon, Greece, Aug. 2013.

[2] A. Borghetti, M. Bosetti, S. Grillo, S. Massucco, C. A. Nucci, M. Paolone and F. Silvestro, Short-Term Scheduling and Control of Active Distribution Systems With High Penetration of Renewable Resources, IEEE Syst. Journal, vol.4, no.3, pp.313-322, Sep. 2010.

[3] G. Valverde and T. Van Cutsem, Model Predictive Control of Voltages in Active Distribution Networks, IEEE Trans. on Smart Grid, vol. 4, no. 4, pp. 2152-2161, Dec. 2013.

[4] H. Soleimani Bidgoli, M. Glavic and T. Van Cutsem, Model predictive control of congestion and voltage problems in active distribution networks, Proc. CIRED conference, paper No 0108, Rome, Italy, Jun. 2014.

[5] P. N. Vovos, A. E. Kiprakis, A. R. Wallace, and G. P. Harrison, Centralized and Distributed Voltage Control: Impact on Distributed Generation Penetration IEEE Trans. on Power Syst., vol. 22, no. 1, pp. 476 - 483, Feb. 2007.

[6] M. J. Dolan, E. M. Davidson, I. Kockar, G. W. Ault, and D. J. McArthur, Distribution Power Flow Management Utilizing an Online Optimal Power Flow Technique, IEEE Trans. on Power Syst., vol. 27, no. 2, pp. 790-799, May 2012.

[7] T. Sansawatt, L. F. Ochoa and G. P. Harrison, Smart Decentralized Control of DG for Voltage and Thermal Constraints Management, IEEE Trans. on Power Syst., vol. 27, no. 3, pp. 1637-1645, Aug. 2012.

[8] J. Hambrick and R. P. Broadwater, Configurable, Hierarchical, ModelBased Control of Electrical Distribution Circuits, IEEE Trans. on Power Syst., vol. 26, no. 3, pp. 1072 - 1079, Aug. 2011.

[9] A. Bernstein,L. Reyes-Chamorro,J.-Y. Le Boudec and M. Paolone A composable method for real-time control of active distribution networks with explicit power setpoints. Part I: Framework, Electric Power Systems Research,vol. 125,pp. 254264, 2015.

[10] J. M. Maciejowski, Predictive Control With Constraints, Englewood Cliffs, NJ: Prentice-Hall, 2002.

[11] S. J. Qin, T. A. Badgwell, A survey of industrial model predictive control technology, Control Engineering Practice, vol. 11, no. 7, pp. 733-764, Jul. 2003.

[12] J. D. Hurley, L. N. Bize, and C. R. Mummert, The adverse effects of excitation system var and power factor controllers, IEEE Transactions on Energy Conversion, vol. 14, no. 4, pp. 16361645, Dec. 1999.

[13] G. Tsourakis, B.M. Nomikos and C. Vournas, Effect of wind parks with doubly fed asynchronous generators on small-signal stability, Electrical Power System Research, vol. 79, no. 1, pp. 190-200, Jan. 2009.

[14] P. Aristidou, S. Lebeau and T. Van Cutsem, Power System Dynamic Simulations Using a Parallel Two-Level Schur-Complement Decomposition, IEEE Transactions on Power Systems, pp. 112, 2015.

[15] A collection of Fortran codes for large scale scientific computation, 2011. [Online]. Available: http://www.hsl.rl.ac.uk 